\title{
Suppression of Jab1 expression inhibits proliferation and promotes apoptosis of AMC-HN-8 cells
}

\author{
PEI-HUA LI ${ }^{1 *}$, LIN WANG $^{2 *}$, YAO-JIE PAN ${ }^{2,3 *}$, MIAO-MIAO SANG ${ }^{4}$, \\ JUN-NIAN ZHENG ${ }^{2,5}$ and DONG-SHENG PEI ${ }^{6}$
}

\author{
${ }^{1}$ Department of Otorhinolaryngology, Affiliated Hospital of Xuzhou Medical University; \\ ${ }^{2}$ Jiangsu Center for The Collaboration and Innovation of Cancer Biotherapy, Xuzhou Medical University, Xuzhou, \\ Jiangsu 221002; ${ }^{3}$ Department of Oncology, The Affiliated Yancheng Hospital of Medicine School of Southeast University, \\ Yancheng, Jiangsu 224001; ${ }^{4}$ Department of Rehabilitation Medicine, Affiliated Hospital of Xuzhou Medical University; \\ ${ }^{5}$ Center of Clinical Oncology, Affiliated Hospital of Xuzhou Medical University; ${ }^{6}$ Department of Pathology, \\ Xuzhou Medical University, Xuzhou, Jiangsu 221002, P.R. China
}

Received June 6, 2016; Accepted October 12, 2017

DOI: $10.3892 / 01.2018 .7963$

\begin{abstract}
Jun activation domain-binding protein-1 (Jab1) is a multifunctional protein involved in cell proliferation and apoptosis, DNA damage and repair and genome stability. In a number of types of human carcinoma, the abnormal expression of Jab1 is associated with poor prognosis, suggesting that Jab1 serves a vital function in tumorigenesis. However, the functional effects and the underlying molecular mechanisms of Jab1 in laryngeal squamous cell carcinoma (LSCC) progression remain poorly understood. The results of the present study demonstrate that downregulating Jab1 expression promotes LSCC apoptosis while inhibiting the proliferation of LSCC cells. Furthermore, Jab1 inhibition results in decreased protein kinase B phosphorylation accompanied by increased caspase-3 cleavage and p53 expression. It has been identified that the increased expression of Jab1 is markedly associated with LSCC progression, therefore Jab1 may be used as a novel target for the treatment of laryngeal cancer.
\end{abstract}

\section{Introduction}

The constitutive photomorphogenesis 9 (COP9) signalosome complex (CSN) is composed of 8 subunits (CSN1-CSN8) and

Correspondence to: Professor Jun-Nian Zheng, Jiangsu Center for the Collaboration and Innovation of Cancer Biotherapy, Xuzhou Medical University, 84 West Huai-Hai Road, Xuzhou, Jiangsu 221002, P.R. China E-mail: jnzheng@xzmc.edu.cn

Professor Dong-Sheng Pei, Department of Pathology, Xuzhou Medical University, 209 Tong-Shan Road, Xuzhou, Jiangsu 221002, P.R. China E-mail: dspei@xzhmu.edu.cn

*Contributed equally

Key words: carcinoma, c-Jun activation domain-binding protein-1, proliferation, apoptosis, $\mathrm{p} 53$ is involved in the development of eukaryotic organisms $(1,2)$. $\mathrm{CSN}$ is a highly conserved protein complex that is involved in the regulation of cullin-RING family of ubiquitin ligases (CRLs) by mediating CRL deneddylation (3). The catalytic activity of CSN is performed by the CSN5 subunit. The Mpr1-Pad1-N (MPN) domain of CSN5 harbors a JAB1-MPN-MOV34 (JAMM) metalloenzyme motif (also known as the MPN+ motif), which is responsible for CRL deneddylation (4). Among the 8 subunits, CSN5 is unique, as it possesses the catalytic center of CSN isopeptidase activity and is also able to stably exist independently of the CSN (5). Although CSN5 was initially considered as a c-Jun coactivator, it is now known to be an integral subunit of the CSN. CSN5 is also referred to as c-Jun activation domain binding-protein-1 (Jab1) (6,7). It has been identified previously that the free form of Jab1 is cytoplasmic and nuclear, whereas CSN-associated Jab1 is mainly nuclear (8). Nevertheless, further studies are required to validate the function of Jab1, as a monomer or as a part of the CSN holocomplex, in the formation and progression of tumors.

Jab1 serves an essential function in cellular proliferation by directly interacting with and functionally regulating the activity and stability of several key intracellular regulatory proteins, including p53, p27, mothers against decapentaplegic homolog 4/7, macrophage migration inhibitory factor and hypoxia-inducible factor-1 $\alpha$ (9-13). Although Jab1 participates in a number of regulatory processes, its function in tumorigenesis remains largely elusive. Indeed, Jab1 has an emerging function in cancer. Jab1 overexpression has been identified in various human malignancies and is inversely associated with a poor prognosis of patients with cancer (14). For example, the increased expression of Jab1 is detected in hepatocellular (15) and thyroid (16) carcinoma, and is associated with a lower survival rate.

Among the various types of head and neck cancer known, laryngeal squamous cell carcinoma (LSCC) remains a common type of cancer. There are $>500,000$ novel cases of LSCC diagnosed annually and the incidence of LSCC has increased in 
the last decade $(17,18)$. It is frustrating that the mortality rate of patients with LSCC has not markedly decreased, regardless of whether the treatment strategy for LSCC has improved (19-21). Therefore, novel strategies and biomarkers are necessary for the treatment and tumor staging of LSCC.

In the present study, the expression levels of Jab1 in laryngeal cancer cells were examined. Furthermore, small interfering RNA (siRNA) was employed to analyze the function of Jab1 in regulating apoptosis and proliferation of laryngeal cancer cells in vitro. Finally, in order to investigate the underlying molecular mechanisms by which Jabl is involved in LSCC, the expression levels of protein kinase B (Akt), phosphorylated (p)-Akt, p53 and cleaved caspase-3 (c-caspase-3) were examined in Jab1-knockdown cancer cells. The results of the present study suggest that Jab1 serves a crucial function in the progression of LSCC and may be a promising therapeutic target in combating LSCC.

\section{Materials and methods}

Cell culture conditions and siRNA transfection. AMC-HN-8 and OME cells were sourced from the Cell Bank, China Academy of Sciences (Shanghai, China) and maintained in Dulbecco's modified Eagle's medium (Gibco; Thermo Fisher Scientific, Inc., Waltham, MA, USA) supplemented with $10 \%$ fetal bovine serum (FBS; Invitrogen; Thermo Fisher Scientific, Inc.) and incubated in the Thermo forma incubator (Thermo Fisher Scientific, Inc.) at $37^{\circ} \mathrm{C}$ with $5 \% \mathrm{CO}_{2}$. The cells were grown to $50 \%$ confluence. Cells were then transfected with three sets of Jab1-specific siRNAs (Shanghai GenePharma Co., Ltd., Shanghai, China) or negative control siRNA (siCtrl; Shanghai GenePharma Co., Ltd.), using siLentFect Lipid reagent (Bio-Rad Laboratories, Inc., Hercules, CA, USA). The quantity of the siRNAs used for transfection was 200 pmol for each $35 \mathrm{~mm}$ culture dish. The target sequences of the Jab1-specific siRNAs used were as follows: Jab1-siRNA1 (si1-Jab1) sense, 5'-CCAGACUAUUCCACUUAAUTT-3' and antisense, 5'-AUUAAGUGGAAUAGUCUGGTT-3'; Jab1-siRNA2 (si2-Jab1) sense, 5'-GGUGAAACCAUGAUC AUUTT-3' and antisense, 5'-UAAUGAUCAUGGUUUCAC CTT-3'; Jab1-siRNA3 (si3-Jab1) sense, 5'-GGACUAAGGAUC ACCAUUATT-3' and antisense, 5'-UAAUGGUGAUCCUUA GUCCTT-3'.

At 4-6 $\mathrm{h}$ after transfection, the culture medium (DMEM) containing the transfection reagent was removed, and fresh medium containing FBS was added. After $48 \mathrm{~h}$ of culture, cells were lysed and used for subsequent experiments.

Western blot analysis. At $48 \mathrm{~h}$ after transfection with each si-Jab1, $1 \mathrm{ml}$ of lysis buffer (Beyotime Institute of Biotechnology, Haimen, China) was added to each dish for 30 min at $4^{\circ} \mathrm{C}$, with occasional shaking. Then, cell lysates were collected into a $1.5 \mathrm{ml}$ tube followed with centrifugation at $16,000 \mathrm{x}$ g for $15 \mathrm{~min}$ at $4^{\circ} \mathrm{C}$. The protein concentration was determined using a bicinchoninic acid assay kit (Pierce; Thermo Fisher Scientific, Inc.). Total proteins were boiled with loading buffer (Vicmed Biotech Co., Ltd., Xuzhou, China) and then loaded (100 $\mu \mathrm{g} /$ lane) and separated by SDS-PAGE $(12.5 \%$ gel) and transferred onto a nitrocellulose membrane. The membranes were then blocked for $2 \mathrm{~h}$ at room temperature in 5\% non-fat milk (BD Biosciences, Franklin Lakes, NJ, USA) suspended in tris-buffered saline with Tween-20 (TBST; $150 \mathrm{mM} \mathrm{NaCl}, 20 \mathrm{mM}$ Tris-HCl, pH 8.0, 0.05\% Tween-20). Following blocking, the membranes were incubated with anti-cleaved caspase-3 (Cell Signaling Technology, Inc., Danvers, MA, USA), anti-Jab1, anti-p53, anti-Akt, anti-p-Akt and anti- $\beta$-actin (Santa Cruz Biotechnology, Inc., Dallas, TX, USA) antibodies at $4^{\circ} \mathrm{C}$ overnight. Membranes were then washed using TBST and incubated with the horseradish peroxidase-conjugated mouse anti-rabbit IgG secondary antibodies (cat no. A2074; 1:20,000; Sigma; Merck KGaA, Darmstadt, Germany) at room temperature for $2 \mathrm{~h}$. The protein bands were visualized with Enhanced Chemiluminescence reagent (Tanon Science and Technology Co., Ltd., Shanghai, China). The densitometric analysis for the quantification of the bands was performed using ImageJ software (version 1.46; National Institutes of Health, Bethesda, MD, USA).

Cell proliferation assay. Cell proliferation was evaluated using a Cell Counting Kit-8 (CCK-8; Beyotime Institute of Biotechnology). In brief, at $48 \mathrm{~h}$ post-transfection, 5,000 cells were seeded in 96-well plates with medium containing $10 \%$ FBS and incubated for 1, 2, 3 and 4 days according to the manufacturer's protocol. Then, $10 \mu \mathrm{l} \mathrm{CCK-8} \mathrm{solution} \mathrm{and}$ $100 \mu \mathrm{l}$ serum-free culture medium (DMEM) were mixed and added to each well, followed by incubation at $37^{\circ} \mathrm{C}$ for $2 \mathrm{~h}$. The absorbance was read at $450 \mathrm{~nm}$ using a spectrophotometer (BioTek Instruments, Inc., Winooski, VT, USA). The experiments were performed in triplicate.

Confocal analysis of Annexin $V$ binding. AMC-HN-8 cells were plated in 6-well plates and transfected with si1-Jab1. si1-Jab1 was selected for this and subsequent experiments as it yielded the most marked decrease in Jab1 expression of all three Jab1-specific siRNAs. Following transfection, treated cells were collected in a $1.5 \mathrm{ml}$ tube and washed twice with phosphate buffered saline. Following washing, cells were labeled with $200 \mu \mathrm{l}$ binding buffer (BD Biosciences) containing $5 \mu \mathrm{l}$ Annexin V-fluorescein isothiocyanate (FITC) and added to $300 \mu \mathrm{l}$ binding buffer containing $5 \mu \mathrm{l}$ propidium iodide (PI) at room temperature in darkness for $5 \mathrm{~min}$. Cells were visualized under a fluorescence microscope (Olympus Corporation, Tokyo, Japan). The software used for analysis was Olympus cellSens Dimension (version 1.0; Olympus Corporation). A total of 3 independent experiments were conducted and 5 horizons were randomly selected from each group, at a magnification of $\mathrm{x} 100$.

Flow cytometric analysis of apoptosis. AMC-HN-8 cells were plated in 6-well plates and transfected with si1-Jab1 for $48 \mathrm{~h}$. The cells were collected and labeled using an Annexin VFITC/PI Apoptosis Detection kit (BD Biosciences), according to the manufacturer's protocol. The apoptotic cell fraction was detected using a FACScan flow cytometer (BD Biosciences). Data were analyzed using ModFit LT 3.0 software (Verity Software House, Inc., Topsham, ME, USA). Living cells (Annexin $\mathrm{V}^{-} \mathrm{FITC}^{-} / \mathrm{PI}^{-}$), early apoptotic cells (Annexin $\mathrm{V}^{-} \mathrm{FITC}^{+} / \mathrm{PI}^{-}$), late apoptotic cells (Annexin V-FITC ${ }^{+} / \mathrm{PI}^{+}$) and necrotic cells (Annexin V-FITC-/PI ${ }^{+}$) were enumerated. 

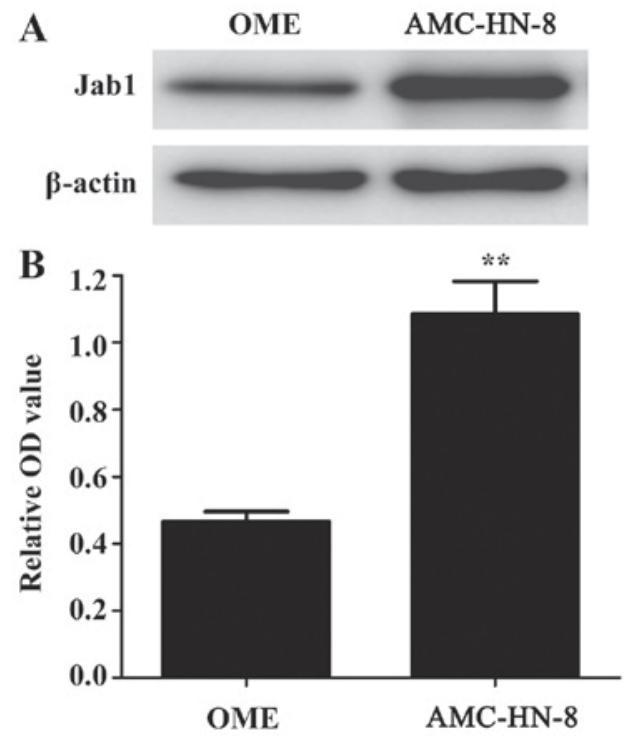

Figure 1. Expression levels of Jab1 protein in normal and cancer cells (A) Western blot analysis of Jab1 protein in OME and AMC-HN-8 cells. (B) Relative intensity determined following densitometric analysis of Jab1 protein in OME and AMC-HN- 8 cells from 3 individual experiments. $\beta$-actin was used as the loading control. Results are expressed as the mean \pm standard deviation. ${ }^{* * *} \mathrm{P}<0.01$ vs. OME. Jab1, c-Jun activation domain-binding protein-1; OD, optical density; OME, oral mucosal epithelial cells.

Statistical analysis. Data were analyzed using SPSS software (version 16.0; SPSS, Inc., Chicago, IL, USA). The relevant data are expressed as the mean \pm standard deviation (SD). Results were analyzed using Student's t-test when only 2 groups were compared, or one-way analysis of variance when $>2$ groups were compared. The post hoc test used was the Student-Newman-Keuls method. $\mathrm{P}<0.05$ was considered to indicate a statistically significant difference.

\section{Results}

Jabl expression is increased in cancer cells. Western blot analysis was used to detect the expression of Jab1 in normal human oral mucosal epithelial (OME) and AMC-HN-8 cells. As presented in Fig. 1, the expression level of Jabl protein was increased in AMC-HN-8 cells compared with OME cells, suggesting that, although Jabl can be expressed in normal cells, its levels are increased in laryngeal cancer cells.

Knockdown of Jabl inhibits cell proliferation and promotes apoptosis. Jab1 expression was knocked down in AMC-HN-8 cells using three different sets of siRNAs targeting Jab1 (si1-Jab1, si2-Jab1 and si3-Jab1). Jab1 protein expression was evaluated using western blot analysis and it was identified that Jab1 was significantly downregulated in si1-Jab1 and si2-Jab1 groups compared with the siCtrl group (Fig. 2). However, si1-Jab1 exhibited the most marked effect in decreasing the expression levels of Jab1 and was therefore used in subsequent experiments.

The effect of Jab1 on carcinoma cell proliferation was investigated. Following transfection of AMC-HN-8 cells with Jab1 siRNA, a CCK-8 assay was performed and a significant decrease in cellular proliferation was identified on days 3 and 4 (Fig. 3). This suggests that loss of Jab1 expression

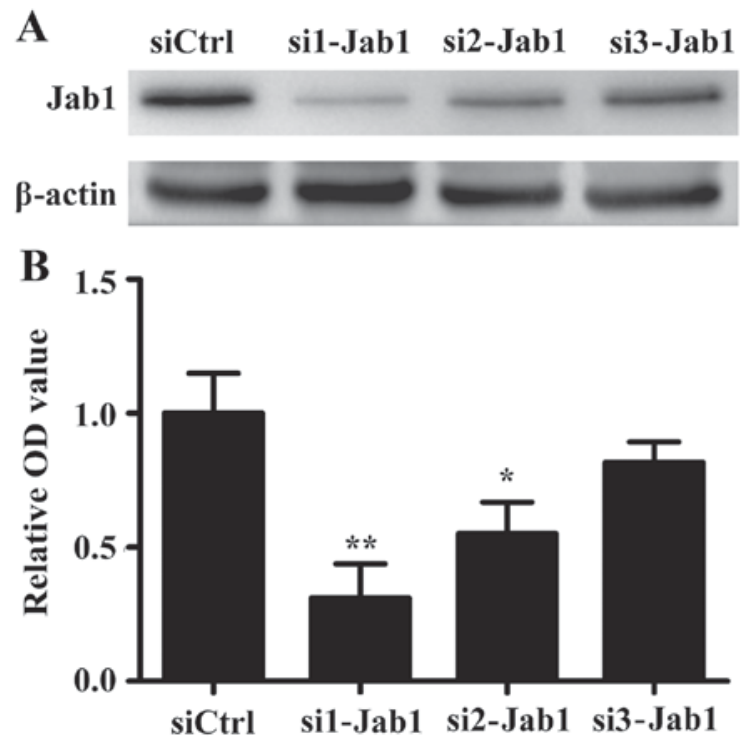

Figure 2.Effect of Jab1 siRNA on the expression levels of Jab1. (A) AMC-HN-8 cells were transfected with siCtrl or with three different sets of Jabl siRNAs: si1-Jab1, si2-Jab1 or si3-Jab1. Following transfection, Jab1 expression was analyzed among the different treatment groups by western blotting. si3-Jab1 group was not significant in the present study, and sil-Jabl was selected for further experiments. (B) Relative intensity obtained after densitometric analysis of Jab1 protein in siCtrl, si1-Jab1, si2-Jab1 and si3-Jab1 treatment groups. $\beta$-actin was used as the loading control. Results are expressed as the mean \pm standard deviation for triplicate experiments. ${ }^{*} \mathrm{P}<0.05 ;{ }^{* *} \mathrm{P}<0.01$ vs siCtrl. Jab1, c-Jun activation domain-binding protein-1; OD, optical density; si-Jab1, Jab1-siRNA; siCtrl, control siRNA; siRNA, small interfering RNA.

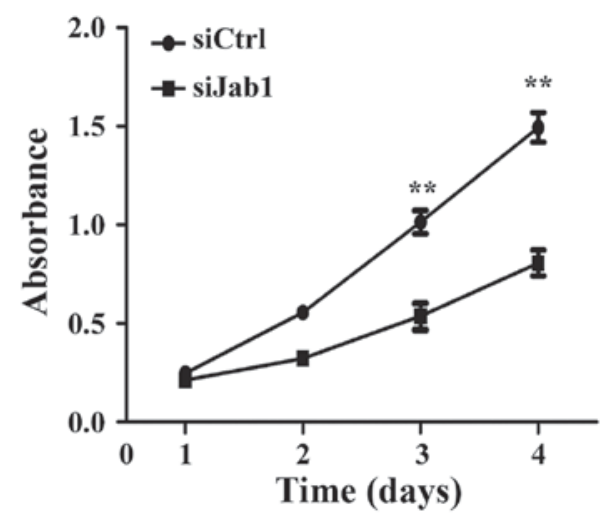

Figure 3. Knockdown of Jab1 suppresses cell proliferation in AMC-HN-8 cells. Proliferation curves from absorbance values obtained on days 1, 2, 3 and 4 in siCtrl and si1-Jab1 treatment groups. Results are expressed as the mean \pm standard deviation for triplicate experiments. ${ }^{* *} \mathrm{P}<0.01 \mathrm{vs}$ siCtrl. Jab1, c-Jun activation domain-binding protein-1; siJab1, Jab1-siRNA1; siCtrl, control siRNA; siRNA, small interfering RNA.

has an inhibitory effect on the proliferation of laryngeal cancer cells.

On the basis of the cell proliferation results, it was investigated whether the loss of Jab1 expression may also have an effect on the apoptosis of laryngeal cancer cells. An Annexin VFITC binding assay and fluorescence microscopy were used to analyze changes in the total apoptotic cells including early apoptotic cells (Annexin V-FITC $+\mathrm{PI}^{-}$) and late apoptotic or necrotic cells (Annexin $\mathrm{V}^{-} \mathrm{FITC}^{+} / \mathrm{PI}^{+}$). It was identified that the si1-Jab1-treated cells exhibited enhanced apoptosis at prophase (Fig. 4A and B) and anaphase (Fig. 4C and D) 
A
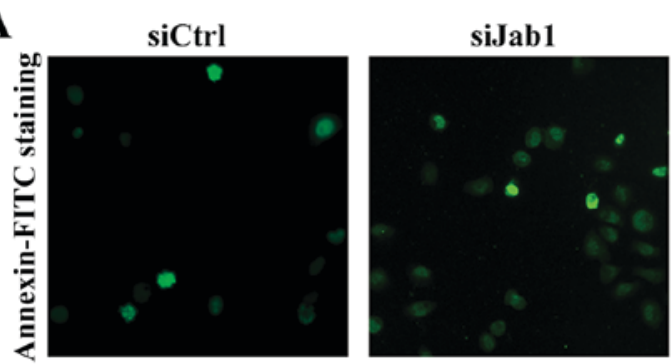

C
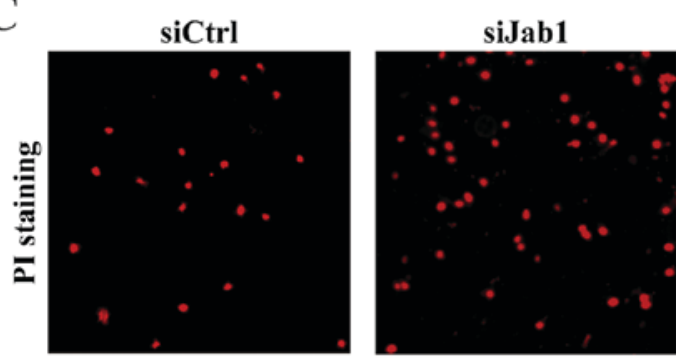

B
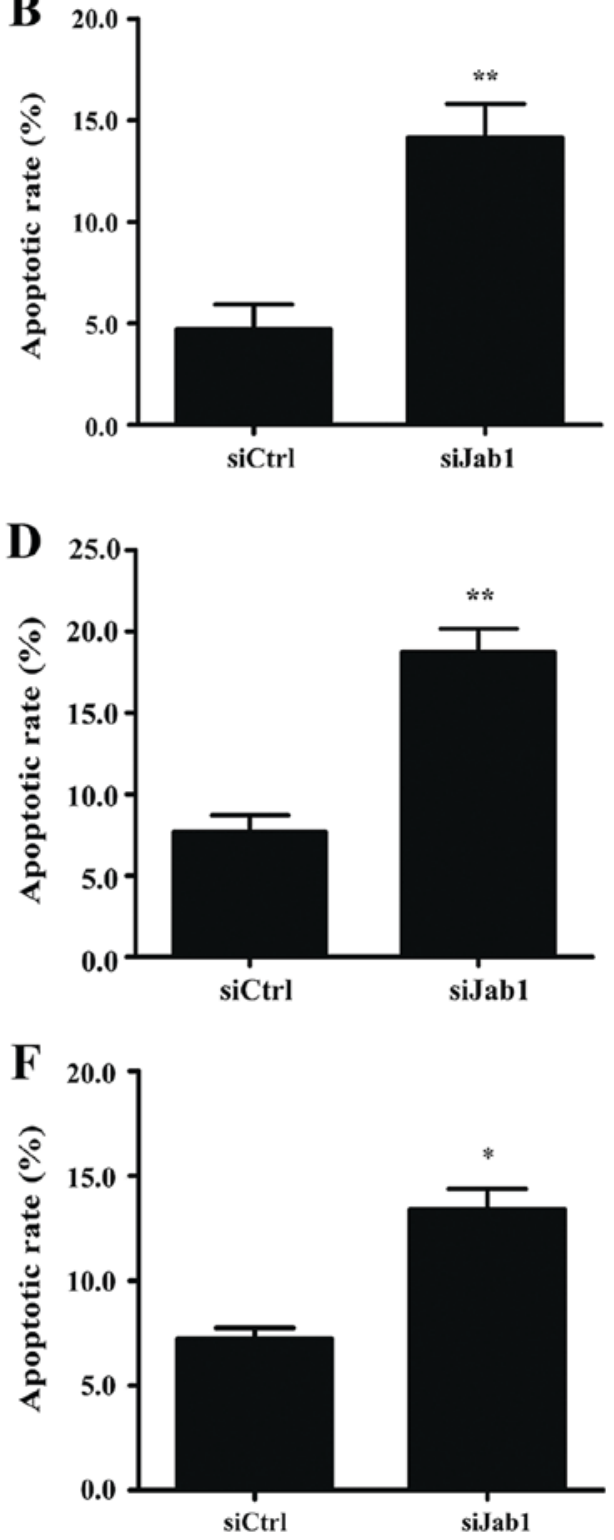

Figure 4. Knockdown of Jab1 facilitates cellular apoptosis in AMC-HN-8 cells. (A) Annexin V-FITC staining identifies cells positive for early stage apoptosis (green). (B) Early apoptotic rate of AMC-HN-8 cells transfected with siCtrl or siJab1. (C) PI staining identified cells positive for late apoptosis or necrosis (red). (D) Late apoptotic rate of AMC-HN-8 cells transfected with siCtrl or siJab1. (E) Dot plots of early and late apoptotic cell populations based on Annexin V-FITC/PI staining in AMC-HN-8 cells transfected with siCtrl or siJab1. (F) Apoptotic rate of AMC-HN-8 cells transfected with siCtrl or siJab1. Results are expressed as the mean \pm standard deviation for triplicate experiments. ${ }^{*} \mathrm{P}<0.05{ }^{* *} \mathrm{P}<0.01$ vs. siCtrl. FITC, fluorescein isothiocyanate; Jab1, c-Jun activation domain-binding protein-1; siJab1, Jab1-siRNA1; PI, propidium iodide; siCtrl, control siRNA; siRNA, small interfering RNA.

compared with the control group. Apoptosis was additionally analyzed between the two groups by flow cytometry. According to the results, it was observed that si1-Jab1 treatment increased the apoptotic cell fraction in AMC-HN-8 cells (Fig. 4E and F). In particular, the Annexin $\mathrm{V}^{-} \mathrm{FITC}^{+} / \mathrm{PI}^{-}$early apoptotic cell fraction accounted for $4.4 \%$ of the cells in the control and $7.3 \%$ of the cells in the si1-Jab1-treated group. Annexin $\mathrm{V}^{-}$FITC ${ }^{+} / \mathrm{PI}^{+}$late apoptotic cells accounted for $2.9 \%$ of the cells in the control and $6.5 \%$ of the cells in the sil-Jab1 group, $48 \mathrm{~h}$ post-transfection. These results suggest that decreasing the expression of Jab1 protein facilitates the apoptosis of laryngeal cancer cells.

Effects of Jabl downregulation on the expression of Akt and p53 protein. To understand the mechanisms involved in
Jab1-mediated apoptosis and proliferation in carcinoma cells, the effect of Jabl deficiency on certain key factors involved in anti-apoptotic pathways and the apoptotic cascade, including Akt, p-Akt, c-caspase-3 and p53, were examined. Western blot analysis revealed that the level of p-Akt expression was downregulated, whereas c-caspase-3 and p53 were upregulated in the Jab1-knockdown group in comparison with the control group (Fig. 5). These results indicate that these proteins may be involved in Jab1-mediated proliferation and apoptosis in AMC-HN-8 cells.

\section{Discussion}

A number of studies have demonstrated that Jabl expression is increased in a number of human malignant tumors (9). Jab1 serves 
A
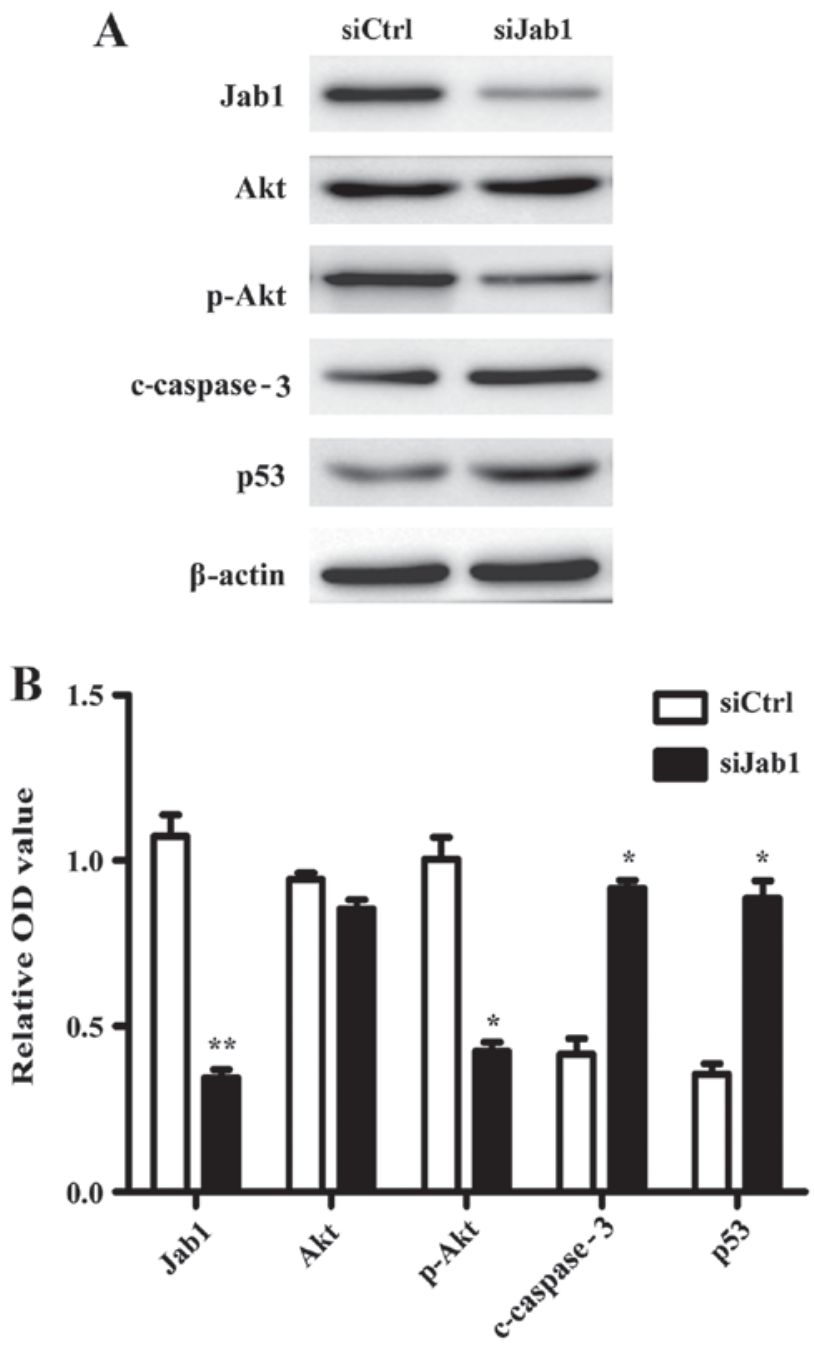

Figure 5. Effects of Jab1 deficiency on the expression of Akt and $\mathrm{p} 53$ proteins (A) Western blot analysis of Akt, p-Akt, c-caspase-3 and p53 expression in AMC-HN-8 cells which were transfected with siCtrl or siJab1. (B) Relative intensity following densitometric analysis of Jab1, Akt, p-Akt, c-caspase-3 and $\mathrm{p} 53$. $\beta$-actin was used as the loading control. Results are expressed as the mean \pm standard deviation for triplicate experiments. ${ }^{*} \mathrm{P}<0.05$; ${ }^{* *} \mathrm{P}<0.01$ vs. siCtrl. Akt, protein kinase B; c-caspase-3, cleaved caspase-3; Jab1, c-Jun activation domain-binding protein-1; p-Akt, phosphorylated Akt; OD, optical density; siJab1, Jab1-siRNA1; siCtrl, control siRNA; siRNA, small interfering RNA.

an intriguing function in the tumorigenic process and increased levels of Jab1 are associated with lymph node metastasis and poor prognosis of several types of human cancer, including LSCC. Jab1 may represent a prognostic indicator of malignant transformation and assists in explaining the biological behavior of a number of cancer cells, including human colorectal cancer cell lines (22) and liver cancer cells (23). It has been reported that $\mathrm{Jab} 1$ acts as a negative regulatory factor of p27 protein and has an association with cell proliferation in LSCC (24). However, the underlying molecular mechanism of Jab1 in LSCC is not known. In the present study, it was identified that AMC-HN-8 cells exhibit increased Jab1 expression levels when compared with OME cells. Additionally, it was demonstrated that downregulation of Jab1 inhibits proliferation and increases the apoptosis of laryngeal carcinoma cells. These results suggest that Jab1 may regulate the biological behavior of the LSCC cells and contribute to the progression of laryngeal carcinoma.
Free-form (non-CSN-associated) Jab1 is located in the cytoplasm and the nucleus, whereas CSN-associated Jab1 is primarily located in the nucleus (25). Jab1 protein is mainly localized in the nucleus in AMC-HN-8 cells (24). The Jab1 PMN domain contains the JAMM motif, which is essential for CSN deneddylation activity. The functional effects of Jab1 depend on the whole CSN assembly and deletion of any CSN subunit may lead to the inactivation of the CSN complex (26).

In the present study the function of Jab1 on apoptosis, a complex biological process (27), during LSCC progression was evaluated. For this purpose, the expression levels of caspase- 3 and p53 were determined in laryngeal cancer cells, with downregulated Jabl expression. Caspase-3 is an effector caspase involved in the apoptotic cascade (28). According to the results of the present study, c-caspase-3 exhibited a significant increase following Jab1 downregulation in AMC-HN-8 cells. Additionally, p53 was identified to be upregulated following si1-Jab1 transfection. p53 is a tumor-inhibiting factor that is able to limit cell proliferation by inducing apoptosis, and a number of apoptosis-related genes are transcriptionally regulated by p53 (29,30). Although c-caspase-3 and p53 are involved in Jab1-mediated apoptosis, it remains to be determined whether there is a connection between p53 and c-caspase-3. According to the results presented in Fig. 5A, p53 and c-caspase- 3 were identified to be upregulated following si1-Jab1 transfection. Therefore, it was hypothesized that there may exist a positive association between p53 and c-caspase-3. Finally, it was identified that the expression level of p-Akt was decreased following si1-Jab1 transfection. On the basis of the significant function of Akt in cellular proliferation (31), it is likely that Jab1 siRNA inhibits cell proliferation via the phosphoinositide 3-kinase/Akt signaling pathway.

In summary, the results of the present study demonstrate that Jab1 serves a crucial function in LSCC tumorigenesis, particularly in cellular proliferation and apoptosis. Jab1 is critical for the regulation of Akt, c-caspase-3 and p53. Further experiments remain to be performed to elucidate the underlying molecular mechanisms by which Jab1 regulates proliferation and apoptosis during tumorigenesis. Jab1 is likely to be a key marker and target for the treatment of LSCC.

\section{Acknowledgements}

The present study was supported by the National Natural Science Foundation of China (grant no. 81572349), Jiangsu Provincial Medical Talent, and the Science and Technology Department of Jiangsu Province (grant nos. BK20130231 and BK20141149).

\section{References}

1. Kapelari B, Bech-Otschir D, Hegerl R, Schade R, Dumdey R and Dubiel W: Electron microscopy and subunit-subunit interaction studies reveal a first architecture of COP9 signalosome. J Mol Biol 300: 1169-1178, 2000.

2. Wei N, Serino G and Deng XW: The COP9 signalosome: More than a protease. Trends Biochem Sci 33: 592-600, 2008.

3. Schwechheimer C and Deng XW: COP9 signalosome revisited: A novel mediator of protein degradation. Trends Cell Biol 11: 420-426, 2001. 
4. Cope GA, Suh GS, Aravind L, Schwarz SE, Zipursky SL, Koonin EV and Deshaies RJ: Role of predicted metalloprotease motif of Jab1/Csn5 in cleavage of Nedd8 from Cul1. Science 298: 608-611, 2002

5. Wei N and Deng XW: The COP9 signalosome. Annu Rev Cell Dev Biol 19: 261-286, 2003.

6. Claret FX, Hibi M, Dhut S, Toda T and Karin M: A new group of conserved coactivators that increase the specificity of AP-1 transcription factors. Nature 383: 453-457, 1996.

7. Chamovitz DA and Segal D: JAB1/CSN5 and the COP9 signalosome. A complex situation. EMBO Rep 2: 96-101, 2001.

8. Kwok SF, Solano R, Tsuge T, Chamovitz DA, Ecker JR, Matsui M and Deng XW: Arabidopsis homologs of a c-Jun coactivator are present both in monomeric form and in the COP9 complex, and their abundance is differentially affected by the pleiotropic cop/det/fus mutations. Plant Cell 10: 1779-1790, 1998.

9. Shackleford TJ and Claret FX: JAB1/CSN5: A new player in cell cycle control and cancer. Cell Div 5: 26, 2010.

10. Winner M, Koong AC, Rendon BE, Zundel W and Mitchell RA Amplification of tumor hypoxic responses by macrophage migration inhibitory factor-dependent hypoxia-inducible factor stabilization. Cancer Res 67: 186-193, 2007.

11. Oh W, Lee EW, Sung YH, Yang MR, Ghim J, Lee HW and Song J: Jab1 induces the cytoplasmic localization and degradation of p53 in coordination with Hdm2. J Biol Chem 281: 17457-17465, 2006.

12. Wan M, Cao X, Wu Y, Bai S, Wu L, Shi X, Wang N and Cao X Jab1 antagonizes TGF-beta signaling by inducing Smad4 degradation. EMBO Rep 3: 171-176, 2002.

13. Tomoda K, Kubota Y, Arata Y, Mori S, Maeda M, Tanaka T, Yoshida M, Yoneda-Kato N and Kato JY: The cytoplasmic shuttling and subsequent degradation of p27Kip1 mediated by Jab1/CSN5 and the COP9 signalosome complex. J Biol Chem 277: 2302-2310, 2002.

14. Kato JY and Yoneda-Kato N: Mammalian COP9 signalosome. Genes Cells 14: 1209-1225, 2009.

15. Hsu MC, Huang CC, Chang HC, Hu TH and Hung WC: Overexpression of Jab1 in hepatocellular carcinoma and its inhibition by peroxisome proliferator-activated receptor $\{$ gamma\} ligands in vitro and in vivo. Clin Cancer Res 14: 4045-4052, 2008.

16. Ahn J, Hong SA, Lee SE, Kim J, Oh YS, Park SJ and Chung YJ: Cytoplasmic localization of Jab1 and p27 Kip1 might be associated with invasiveness of papillary thyroid carcinoma. Endocr J 56: 707-713, 2009

17. Jemal A, Siegel R, Ward E, Hao Y, Xu J and Thun MJ: Cancer statistics, 2009. CA Cancer J Clin 59: 225-249, 2009.
18. Liu XK, Li Q, Xu LH, Hu LJ, Liao WG, Zhang XR, Liu ZM, Wu D and Zeng MS: Expression and clinical significance of SIAH in laryngeal squamous cell carcinoma. Med Oncol 30: 485, 2013

19. Boyle P and Ferlay J: Cancer incidence and mortality in Europe, 2004. Ann Oncol 16: 481-488, 2005.

20. Ferlay J, Parkin DM and Steliarova-Foucher E: Estimates of cancer incidence and mortality in Europe in 2008. Eur J Cancer 46: 765-781, 2010.

21. Li JJ, Yang XM, Wang SH and Tang QL: Prognostic role of epidermal growth factor-like domain 7 protein expression in laryngeal squamous cell carcinoma. J Laryngol Otol 125: $1152-1157,2011$

22. Schütz AK, Hennes T, Jumpertz S, Fuchs S and Bernhagen J: Role of CSN5/JAB1 in Wnt/ $\beta$-catenin activation in colorectal cancer cells. FEBS Lett 586: 1645-1651, 2012.

23. Lee YH, Judge AD, Seo D, Kitade M, Gómez-Quiroz LE, Ishikawa T, Andersen JB, Kim BK, Marquardt JU, Raggi C, et al: Molecular targeting of CSN5 in human hepatocellular carcinoma: A mechanism of therapeutic response. Oncogene 30: 4175-4184, 2011.

24. Dong Y,SuiL, Watanabe Y, YamaguchiF, Hatano N and Tokuda M: Prognostic significance of Jab1 expression in laryngeal squamous cell carcinomas. Clin Cancer Res 11: 259-266, 2005.

25. Tomoda K, Kubota Y and Kato J: Degradation of the cyclin-dependent-kinase inhibitor p27Kip1 is instigated by Jab1. Nature 398: 160-165, 1999.

26. Adler AS, Littlepage LE, Lin M, Kawahara TL, Wong DJ, Werb Z and Chang HY: CSN5 isopeptidase activity links COP9 signalosome activation to breast cancer progression. Cancer Res 68: 506-515, 2008.

27. Thompson CB: Apoptosis in the pathogenesis and treatment of disease. Science 267: 1456-1462, 1995.

28. Porter AG and Jänicke RU: Emerging roles of caspase-3 in apoptosis. Cell Death Differ 6: 99-104, 1999.

29. Shen Y and White E: p53-dependent apoptosis pathways. Adv Cancer Res 82: 55-84, 2001.

30. Abu-Qare AW and Abou-Donia MB: Biomarkers of apoptosis: Release of cytochrome c, activation of caspase-3, induction of 8-hydroxy-2'-deoxyguanosine, increased 3-nitrotyrosine, and alteration of p53 gene. J Toxicol Environ Health B Crit Rev 4: 313-332, 2001.

31. Cicenas J: The potential role of Akt phosphorylation in human cancers. Int J Biol Markers 23: 1-9, 2008. 